\title{
High-birefringent photonic crystal fiber
}

Libori, Stig E. Barkou; Broeng, Jes; Knudsen, Erik; Bjarklev, Anders Overgaard; Simonsen, Harald R.

\section{Published in:}

Proceedings on Optical Fiber Communication Conference and Exhibit

Link to article, DOI:

10.1109/OFC.2001.927347

Publication date:

2001

Document Version

Publisher's PDF, also known as Version of record

Link back to DTU Orbit

Citation (APA):

Libori, S. E. B., Broeng, J., Knudsen, E., Bjarklev, A. O., \& Simonsen, H. R. (2001). High-birefringent photonic crystal fiber. In Proceedings on Optical Fiber Communication Conference and Exhibit (Vol. 2)

https://doi.org/10.1109/OFC.2001.927347

\section{General rights}

Copyright and moral rights for the publications made accessible in the public portal are retained by the authors and/or other copyright owners and it is a condition of accessing publications that users recognise and abide by the legal requirements associated with these rights.

- Users may download and print one copy of any publication from the public portal for the purpose of private study or research.

- You may not further distribute the material or use it for any profit-making activity or commercial gain

- You may freely distribute the URL identifying the publication in the public portal 


\title{
High-birefringent photonic crystal fiber
}

\author{
Stig Barkou Libori, Jes Broeng, Erik Knudsen, Anders \\ Bjarklev \\ Research Center COM, Technical University of Denmark, Building $345 \varnothing$, \\ DK-2800 Lyngby, Denmark \\ Harald R. Simonsen \\ Crystal Fibre A/S, Priorparken 878, 2605 Brøndby, Denmark \\ seb@com.dtu.dk
}

\begin{abstract}
A highly birefringent Photonic Crystal Fiber design is analysed. Birefringence up to $10^{-3}$ is found. Random fluctuations in the cladding design are analysed, and the fiber is found to be a feasible polarization maintaining fiber.

(C) 2000 Optical Society of America.

OCIS codes: (260.1440) Birefringence; (060.2400) Fiber properties
\end{abstract}

Introduction: Photonic Crystal Fibers, $\mathrm{PCFs}$, have generated tremendous interest during the last few years $[1,2,3]$. The cladding of a PCF consist of a large number of air-holes embedded within a silica background. The fibres are invariant in the longitudinal direction. Usually, as is the case for the PCFs investigated in this paper, the core-region is formed by omitting the central air-hole, thereby creating a silica core-region with a higher index than the effective index of the surrounding air-filled cladding. Such PCFs are especially renowned by their ability to support only a single degenerate mode in the entire wavelength range [1], if the air-filling fraction of the cladding is not to large.

In this paper we explore the attractive possibility of employing PCFs as polarisation maintaining fibres. This is specifically relevant as more and more optical processing becomes necessary in optical networks, where enhanced optical functionality is required. We find that a high degree of birefringence is indeed possible in PCFs. To be able to successfully maintain the polarization of light transmitted by the fibre, it is necessary to be able to control the magnitude of birefringence one can expect in PCFs due to small production deviations away from a perfect crystalline symmetry of the cladding. To our knowledge, this is the first theoretical investigation of the magnitude of intentional birefringence compared to non-intentional birefringence in PCFs.

Intentionally introduced birefringence: For single mode PCFs (as are the fibres investigated in this paper), the guided mode consists of two degenerate polarizations. To deliberately introduce birefringence into this guided mode, we have enlarged two of the central air-holes, as shown in Fig.1. Fig.1 also defines the lattice constant $\Lambda_{1}$ and the cladding hole diameter $D_{1}$. The two enlarged cladding holes have diameter $D_{2}$. To further increase the birefringence, the two enlarged cladding holes are placed at a reduced distance, $\Lambda_{2}$ from the center of the core. As seen in Fig. 1 this design breaks the 60 degrees symmetry usually present in PCFs, and instead introduces an elongated core region with 180 degrees symmetry. We expect such a core-region to yield the highest degree of birefringence (this is in complete analogy with standard optical fibres). The particular structure analysed here, has $D_{1}=0.45 \Lambda_{1}$ and $D_{2}=0.75 \Lambda_{1}$, while $\Lambda_{2}=0.8 \Lambda_{1}$. All calculations, in this paper, were performed with a plane-wave method [4], using a supercell with the area of 48 simple cells. We use $256 \times 256$ plane waves for each polarization.

The geometrical size of the core-region of this PCF design is uniquely determined by $\Lambda_{1}$. By assuming that material dispersion only has limited influence on the birefringence (we have used 1.45 as the refractive index of silica in our calculations), it becomes possible to illustrate the effect of changing the wavelength and or $\Lambda_{1}$ in a single plot. This is illustrated in Fig.2a, where the curve 1 shows the magnitude of the birefringence of this fibre as a function of the normalized frequency, $\frac{\Lambda_{1}}{\lambda}$, where $\lambda$ is the free space wavelength. Working with this normalized frequency also aids the design process, since one may easily find the necessary $\Lambda_{1}$ for a desired combination of birefringence and wavelength.

From Fig.2a we conclude that a birefringence of more than $10^{-3}$ is possible, when the structure-size $\Lambda_{1}$ is smaller than twice the free-space wavelength. It is worth noticing, that $\frac{\Lambda_{1}}{\lambda}=3$ corresponds to a core-diameter of 5-6 microns! We conclude, that it is possible to design PCFs with high birefringence, while maintaining a realistic core-size. 


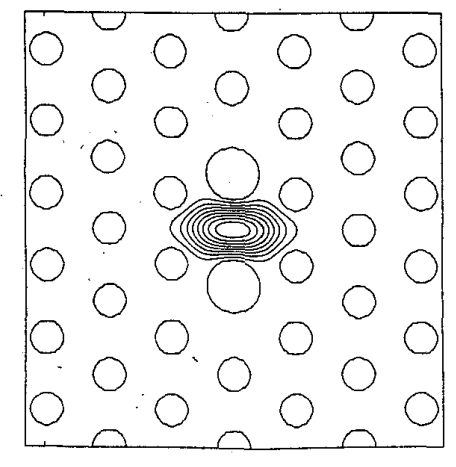

Fig. 1. High-birefringent PCF design. The birefringence arises due to the two enlarged air-holes near the center of the fiber, which breaks the 60 degrees symmetry. The field solution shown is for $\frac{\Lambda_{1}}{\lambda}=3$, where the birefringence is $5 \times 10^{-4}$

Non-intentional birefringence: The subject of birefringence in PCFs arising because of imperfections of the cladding structure has not yet been investigated. It seems reasonable to assume that the non-intentional birefringence will be highest, when the effective-index difference between the cladding and the core is great. That is, we expect the highest degree of non-intentional birefringence for large air-holes compared to, the wavelength, or in other words: birefringence is expected to increase with wavelength and with air-filling fraction. Furthermore, we expect birefringence to be most profound when large deviations from the crystalline cladding design appear near the core-region, and when the cladding deviations have a close-to-180-degrees symmetry (as was the case with the design for deliberate birefringence investigated above).

We here numerically investigate the effect of cladding imperfections in PCFs in the following way: We demand that the cladding air holes be perfectly circular. However, instead of assuming each air-hole to have $D=0.45 \Lambda$, we allow the hole diameter to be randomly (evenly) distributed in the interval $D=0.36 \Lambda$ to $D=0.54 \Lambda$. Notice that we now assume $\Lambda=\Lambda_{1}=\Lambda_{2}$, and $D=D_{1}=D_{2}$. To find the maximal birefringence, which can be caused by diameter variations of the cladding structure, we furthermore demand that the deviations have 180 degrees symmetry. The resulting maximal birefringence found, is shown by curve 2 in Fig.2a. As expected the maximal birefringence is large for small core PCFs (i.e., well above $10^{-4}$ when $\Lambda$ is as small as the wavelength), while it is significantly lower for large-core PCFs (i.e., below $10^{-5}$ when $\Lambda>5 \lambda$ ).

We have also calculated the effect of fixing the cladding air-hole diameters at $D=0.45 \Lambda$, while allowing the positioning of the air-holes to randomly deviate from the ideal cladding structure. Specifically, the cladding hole centers are distributed evenly within discs with a radius equal to $0.03 \Lambda$. Again, we also require the structure to have inversion symmetry to maximize the birefringence found. The largest birefringence found is depicted by curve 3 in Fig.2a. Now, $\Lambda_{1}$ is the average distance between air-holes. We notice that the chosen variation in position, yields a maximal calculated birefringence close to that found when varying the hole-diameter. Finally, curve 4 in Fig.2a shows the effect of varying both cladding hole diameter and position at the same time. A typical example of the placing and size of the air-holes for this last calculation is depicted in Fig.2b.

Despite the large birefringence found by deliberately choosing an asymmetric core-design, it seems difficult to obtain deliberate birefringence, which is orders of magnitude larger, than the birefringence arising from accidental deviations from the perfect cladding structure with 60 degrees rotational symmetry. A few remarks should, however, be taken into account, before reaching a conclusion about the feasibility of PCFs as polarization maintaining fibres. First of all, we have assumed all deviations from the perfect cladding structure to have 180 degrees symmetry. This is unlikely to be the case for a real PCF, and we may, therefore, conclude that accidental birefringence will be smaller in real fibres, than what is indicated by curve 4 in Fig.2a. Secondly, as seen in Fig.2b the deviations from the perfect cladding structure are quite large in our calculations. Curve 5 in Fig.2a shows the maximally calculated birefringence, when the deviations are smaller (The diameter varies from 0.44 to 0.46 and the radius of the 'discs' is $0.01 \Lambda$ ). Thirdly, Fig.2a only shows the maximal calculated value of birefringence. In the computations, several runs yielded birefringence 

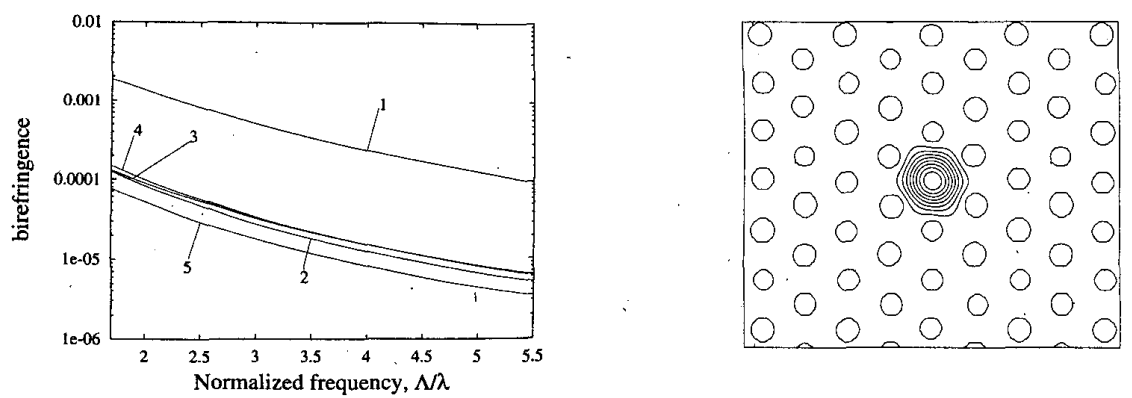

Fig. 2. a) Birefringence in PCFs. The design shown in Fig.1 has the birefringence shown by curve 1. Curves 2 through 5 shows accidental birefringence from random variations in the cladding hole size, the positioning of the holes, or both as explained in the text. b) Example of a typical PCF with random cladding fluctuations. A field solution for $\frac{\Lambda}{\lambda}=3$ is included

up to ten times lower, than the birefringence values depicted in Fig.2a.

Furthermore, keeping in mind that Fig.2a exaggerates the birefringence in real PCFs (due to the inversion symmetry applied in the calculations), we conclude that it is indeed possible to make polarization maintaining PCFs. One sub-conclusion is that it is imperative to be able to accurately calculate the accidental birefringence in real PCFs. Furthermore, a good control of the deviations from a perfect cladding structure is mandatory.

Conclusion: We have analysed a PCF designed to be highly birefringent, and found birefringence of the order of $10^{-3}$ to be realistic in PCFs. However, the birefringence arising from accidental variations in positioning and size of the cladding air-holes, was found to be quite high itself (up to $10^{-4}$, for the quite large random hole-variations analysed here- The hole diameters varied by $+-20 \%$, while the cladding hole centers were randomly displaced by up to $3 \%$ of the typical inter-hole distance). It was argued that it should be possible to manufacture PCFs with significantly lower values of accidental birefringence, thereby making it feasible to benefit from the very high deliberate birefringence, which is possible in PCFs. By 'using numerical tools to identify PCFs with low values of accidental birefringence, it should be possible to create PCFs suitable as polarization maintaining fibers.

\section{References}

1. J.C. Knight, T.A. Birks, P. St.J. Russell, and D.M. Atkin. All-silica single-mode optical fiber with photonic crystal cladding. Optics Letters, 21(19):1547-9, Oct. 1996.

2. S.E. Barkou, J. Broeng, and A. Bjarklev. Novel silica/air photonic crystal fiber allowing waveguiding by the true photonic bandgap effect. Optics Letters, 21:1547-1549, 1999.

3. T. M. Monro, N. G. R. Broderick Bennett, and Richardson D. J. Holey fibers with random cladding distributions. Optics letters, 25(4):206-8, February 2000.

4. R.D. Meade, A.M. Rappe, K.D. Brommer, J.D. Joannopoulos, and O.L. Alerhand. Accurate theoretical analysis of photonic band-gap materials. Physical Review B, 48(11):8434-7, Sept. 1993. 